\title{
Randomized clinical trial on the survival of lithium disilicate posterior partial restorations bonded using immediate or delayed dentin sealing after 3 years of function
}

\author{
van den Breemer, C R G ; Cune, M S ; Özcan, M ; Naves, L Z ; Kerdijk, W ; Gresnigt, M M M
}

\begin{abstract}
OBJECTIVES The survival and success rate and the quality of survival of partial ceramic restorations bonded employing Immediate (IDS) or Delayed Dentin Sealing (DDS) in vital molar teeth were evaluated in a randomized clinical trial with within-subject comparison study. MATERIALS AND METHODS 30 patients received two lithium disilicate ceramic (IPS-e.max press, Ivoclar Vivadent) partial restorations on vital first or second molar teeth $(\mathrm{N}=60)$. The two teeth randomly received either IDS (test group, $\mathrm{n}=30$ ) or DDS (control group, $\mathrm{n}=30$ ). Partial ceramic restorations were luted (Variolink Ultra, Ivoclar Vivadent) two weeks after preparation. Evaluations were performed at 1 week, 12 months and 36 months post-operatively, using qualitative (FDI) criteria. Representative failures were evaluated microscopically (SEM) and by means of simplified qualitative fractography analysis. RESULTS One absolute failure occurred in the DDS group due to (secondary) caries. The overall survival rate according to Kaplan-Meier after 3 years was $98.3 \%$ (FDI criteria score $1-4, \mathrm{n}=59$ ) and the overall success rate was $85 \%$ (FDI criteria score $1-3, \mathrm{n}=51$ ), with no significant difference between restorations in the IDS and DDS group ( $\mathrm{p}=0.32$; Kaplan-Meier, Log Rank (Mantel-Cox), CI = 95\%). For the quality of the survival, no statistically significant differences were found between IDS and DDS ( $\mathrm{p}=0.7$; Kaplan-Meier, Log Rank (Mantel-Cox), CI = 95\%) restorations on any follow-up timepoints for any of the FDI criteria (Wilcoxon, McNemar, p > 0.05). CONCLUSION Adhesively luted partial ceramic restorations in vital molar teeth have a good prognosis, however IDS did not show any differences in success and survival rates after 3 years of function.
\end{abstract}

DOI: https://doi.org/10.1016/j.jdent.2019.02.001

Posted at the Zurich Open Repository and Archive, University of Zurich

ZORA URL: https://doi.org/10.5167/uzh-183058

Journal Article

Accepted Version

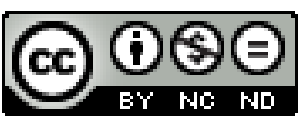

The following work is licensed under a Creative Commons: Attribution-NonCommercial-NoDerivatives 4.0 International (CC BY-NC-ND 4.0) License.

Originally published at:

van den Breemer, C R G; Cune, M S; Özcan, M; Naves, L Z; Kerdijk, W; Gresnigt, M M M (2019). Randomized clinical trial on the survival of lithium disilicate posterior partial restorations bonded using immediate or delayed dentin sealing after 3 years of function. Journal of Dentistry, 85:1-10. 
DOI: https://doi.org/10.1016/j.jdent.2019.02.001 


\section{Randomized Clinical Trial on the Survival of Lithium Disilicate Posterior Partial Restorations Bonded Using Immediate or Delayed Dentin Sealing Under 3 Years of Function}

\section{CRG van den Breemer* $\bullet$ MMM Gresnigt $\bullet$ M Özcan • LZ Naves • W Kerdijk • MS Cune}

*Carline van den Breemer, DDS, Research Fellow, University of Groningen, University Medical Center Groningen, Center for Dentistry and Oral Hygiene, Department of Fixed and Removable Prosthodontics and Biomaterials, Groningen, The Netherlands

Marco Gresnigt, DDS, PhD, University Medical Center Groningen, Center for Dentistry and Oral Hygiene, Department of Fixed and Removable Prosthodontics and Biomaterials, Groningen, The Netherlands

Martini Hospital, Department of Prosthodontics and Special Care, Groningen, The Netherlands Mutlu Özcan, DDS, PhD, Professor, University of Zurich, Division of Dental Materials, Center for Dental and Oral Medicine, Clinic for Fixed and Removable Prosthodontics and Dental Materials Science, Zurich, Switzerland

Lucas Zago Naves, DDS, PhD, University of Groningen, University Medical Center Groningen, Center for Dentistry and Oral Hygiene, Department of Fixed and Removable Prosthodontics and Biomaterials, Groningen, The Netherlands

Wouter Kerkdijk, PhD, Hanze University of Applied Sciences, Department of Education and Research, Groningen, The Netherlands

Marco Cune, DDS, PhD, Professor, University of Groningen, University Medical Center Groningen, Center for Dentistry and Oral Hygiene, Department of Fixed and Removable Prosthodontics and Biomaterials, Groningen, The Netherlands; St. Antonius Hospital Nieuwegein, Department of Oral Maxillofacial Surgery, Prosthodontics and Special Dental Care, Nieuwegein, The Netherlands; University Medical Center Utrecht, Department of Oral Maxillofacial Surgery, Prosthodontics and Special Dental Care, Utrecht, The Netherlands 
Running title: Immediate and delayed dentin sealing effect on survival of partial restorations

\section{*Corresponding author:}

Carline R.G. van den Breemer

University Medical Centre Groningen

Centre for Dentistry and Oral Hygiene

Department of Fixed and Removable Prosthodontics

Antonius Deusinglaan 1

9713 AV, Groningen, The Netherlands

Tel: +31503632673

e-mail: c.r.g.van.den.breemer@umcg.nl (C.van den Breemer) 


\section{SUMMARY}

The survival and the quality of survival of partial ceramic restorations bonded employing Immediate (IDS) or Delayed Dentin Sealing (DDS) in vital molar teeth was evaluated in a randomized clinical trial with within-subject comparison study. Between December 2013 and March 2016, a total of 30 patients (13 women, 17 men; mean age: 54 years) received two lithium disilicate ceramic (IPS-e.max press, Ivoclar Vivadent) partial restorations on vital first or second molar teeth $(\mathrm{N}=60)$. Partial preparations were performed on all teeth and the two teeth randomly received either IDS (test group, $n=30$ ) or DDS (control group, $\mathrm{n}=30$ ). Partial ceramic restorations were luted (Variolink Ultra, Ivoclar Vivadent) two weeks after preparation. Evaluations were performed at 1 week, 12 months and 36 months postoperatively, using qualitative criteria. Restorations with flaws were evaluated using SEM. Success rate of the restorations after 36 months was $83.3 \%$ (Kaplan-Meier), with no significant difference between restorations in the IDS and DDS group. Nine restorations presented with shortcomings, 5 with DDS and 4 with IDS, leading to an overall survival rate of $98.3 \%$. No statistically significant differences were found between IDS and DDS restorations on any follow-up timepoints for any of the Hickel criteria. One absolute failure occurred in the DDS group due to (secondary) caries. Adhesively luted partial ceramic restorations in vital molar teeth have a good prognosis but do not benefit from IDS with respect to success and survival rates after 3 years of function.

Key words: Adhesion, Immediate Dentin Sealing, Lithium Disilicate, Randomized Clinical Trial, Partial Restorations, Survival 


\section{INTRODUCTION}

Biomechanically or aesthetically compromised teeth can be restored with partial ceramic indirect restorations. Due to advances in adhesive technologies and ceramic materials it is possible to restore teeth at a limited biological price saving sound tooth tissue. The durability of these partial ceramic restorations relies heavily on the adhesive strength of the resin luting cement to the ceramic restoration and to the tooth surface but also on the ceramic material that is used.

Currently numerous ceramic materials are available for fabricating indirect partial restorations. 1,2 Glass ceramics comprise a vitreous and crystalline phase in which a glassy matrix could be etched optimizing the adhesive strength of these materials. 3-5 In contrast, crystalline ceramics, alumina and zirconia, have minimal or practically no vitreous phase. 3,5 These materials differ in mechanical properties which raises the question what material is best suited for the heavily loaded posterior region. A recent meta-analysis on this subject 3 indicated that the type of ceramic material (feldspathic porcelain vs. glass-ceramic), study design (retrospective vs. prospective), followup time (5 vs. 10 years), and study setting (university vs. private clinic) did not affect the survival rate. Estimated survival rates for glass-ceramics and feldspathic porcelain varies between $92 \%$ and $95 \%$ at 5 years and $91 \%$ at 10 years. Failures were related to fractures (4\%), endodontic complications (3\%), secondary caries (1\%) and debonding (1\%). 3 However, long-term data comparing survival and success of various types of all-ceramic crowns are lacking. 6

Adhesion to dentin in particular remains a clinical challenge in clinical dentistry to date. Immediate Dentin Sealing (IDS) has been suggested as an alternative to conventional adhesive luting, also referred to as Delayed Dentin Sealing (DDS). 7-13 With IDS, a thin layer of adhesive resin is applied immediately after tooth preparation and prior to impression taking, whereas with DDS, the adhesive resin layer is applied just before luting the restoration. IDS has been extensively studied and significantly improved over the years with positive results with respect to bond strength, gap 
formations, bacterial leakage, and post-cementation hypersensitivity. 7-10,13-22 However, randomized controlled trials on IDS need to be performed, and consequently it is unknown if IDS is a beneficial procedure, preventing failures in partial indirect restorations. 14

Therefore, the objective of this study is to evaluate the survival and the quality of survival of lithium disilicate posterior partial restorations bonded using immediate or delayed dentin sealing over a 3-year follow up period. The tested null hypotheses were that there would be no significant differences in success and survival rate and the quality of survival between partial indirect ceramic restorations bonded with either IDS or DDS.

\section{METHODS AND MATERIALS}

\section{Study Design}

Between December 2013 and May 2016, a total of 30 patients (13 women, 17 men; mean age: 54 years) with an indication for two indirect partial ceramic restorations on first or second vital molar teeth were recruited. The inclusion criteria were the following: physically and psychologically able to tolerate conventional restorative procedures; good oral hygiene; presence of intact buccal wall of the tooth; normal response on cold test; possibility to apply rubber dam; presence of the antagonistic tooth; and willingness of the patient to return for follow-up examinations.

The two teeth randomly received either IDS (test group, $n=30$ ) or DDS (control group, $n=30$ ) through randomization software (www.randomizer.org). Hence, the study can be characterized as a randomized controlled, single blind clinical trial with within-subject comparison. A consort flow chart showing the enrollment, intervention allocation, follow-up, and data analysis is presented in Figure 1. The study was approved by the Medical Ethics Committee of the University Medical Center Groningen, The Netherlands (ABR number: NL 45130) and registered in the Clinical Trial Register of the US National Library of Medicine (NCT03443583). All patients were provided with informed consent. Distribution of restorations and extension of the restorations are presented in Table 1. 


\section{Tooth preparation}

The brands, types, manufacturers, chemical compositions and batch numbers of the main materials used in this study are listed in Table 2. After isolating the teeth with rubber dam (Hygenic Dental dam, Coltène/Whaledent Inc., Ohio, USA) the existing restorations were removed. Students executed tooth preparation and luting of the restoration in their first, second or third year of their dentistry masters' closely supervised by one dentist. The outline configuration was a butt shoulder, prepared using diamond burs and specific inserts for inlay preparations in an ultrasonic handpiece (SONICflex prep ceram, KaVo GmbH, Biberach/Riss, Germany). All internal angles were smoothened to reduce stress concentration. The cusps were covered $(1.5 \mathrm{~mm})$ if the remaining tooth structure wall was less than 2 $\mathrm{mm}$ thick from its occlusal aspect or when the outline of the restoration would be in an area with static or dynamic antagonist contacts. Slight divergence with an angle of 100 to $120^{\circ}$ between the proximal cavity walls and the prospective proximal inlay surfaces were provided. The dental technician blocked out any incidental undercuts in the teeth that were allocated to the control group (DDS), the remaining cases were compensated for by the IDS.

The teeth on the test group received IDS (Clearfil SE Primer and Adhesive, Clearfil Majesty Flow, Kuraray) immediately after exposure of dentin (table 3a). Electrosurgery was performed in cases where retraction of the gingiva was required for proper impression making. Impressions were made using a silicone impression material (Heavy and Ultra Light body Aquasil, Dentsply, Mildford, USA) using an individually designed acrylic impression tray. Temporary restorations were then made chairside using a chemically polymerized resin material (Protemp, 3M ESPE, Neuss, Germany) and cemented using polycarboxylate cement (Durelon, 3M ESPE, Minn, USA).

\section{Luting}

One dental technician fabricated all lithium disilicate restorations (IPS e.max press, Ivoclar Vivadent, Schaan, Liechtenstein) following manufacturer instructions. Restorations were glazed at low 
temperatures applied to the restoration after construction (FLUO IPS e.max Ceram Glaze paster, Ivoclar Vivadent). Two weeks after preparation, the temporary cement was removed from the teeth with an ultrasonic tip and a scaler. The sequence of the different tooth conditioning and restoration procedures, before luting are presented in Tables $3 a-b$ and 4 . The adhesive procedure differed between the test and control group, as outlined in these tables. All the partial restorations were luted using a heated $\left(55^{\circ} \mathrm{C}\right)$ dual-polymerized luting composite (Variolink Ultra, Ivoclar Vivadent). Restorations were placed initially under slight pressure where the excess material was removed immediately from the margins with a probe, a scaler and dental floss (Oral-B, Rotterdam, The Netherlands). After increasing the pressure, the final excess composite was manipulated against the tooth in order to prevent marginal gaps. The restorations were photo-polymerized $\left(>1.000 \mathrm{~mW} / \mathrm{cm}_{2}\right.$, Bluephase Style, Ivoclar Vivadent) for 40 seconds from 3 sides and this was repeated after the application of glycerin gel (K-Y Johnson \& Johnson, Sezanne, France). Occlusion and articulation was

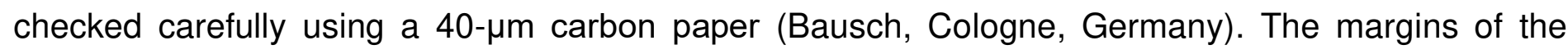
restorations were finished using a scaler and an ultrasonic device (EVA-handpiece 7LP in combination with a $61 \mathrm{LG}, \mathrm{KaVo} \mathrm{GmbH}$ ) and polished using ceramic polishers (CeraGloss blue and yellow, Edenta, Argau, Switzerland). Intra-oral radiograph was then made in order to check for excess composite in the cervico-approximal region.

\section{Evaluation}

Restorations were evaluated at baseline ( 1 week after luting of the restorations) and thereafter at 12 months and 36 months. One observer evaluated the restorations according to the Hickel criteria 23 calibrated by the e-calib web-based training (www.e-calib.info). The Hickel critera are used to measure the quality of survival and determining the success or survival of a restoration. Restorations without any major adverse effects scored 1-3 on Hickel criteria and were considered as a success. Restorations with a score 4 on any of the Hickel criteria were considered repairable failures and considered to have survived. Restorations with a score 5 on Hickel criteria were non-reparable 
failures and were considered as absolute failures. With Hickel criteria the most severe score would prevail. The proximal contact points were checked by passing waxed dental floss (Johson\&Johnson, Sezanne) through the interdental space. Restorations were visually (2.3x magnification loops, Examvision, Rotterdam, The Netherlands) inspected with a dental mirror and probe. Patients were instructed to call if any kind of failure occurred. Digital photographs (1:1) and intra-oral radiographs were made after placement of the restorations and during follow-up sessions.

\section{SEM (Scanning Electron Microscopy)}

In case of any failure an impression (Ultra Light and Heavy body Aquasil, Dentsply, Mildford, USA) was made from the failure site after cleansing the surface with absorbent paper and hypochlorite 0.5\%. Impressions were poured with cold mounting epoxy resin (EpoxyCure, Buehler, IL, USA) which was subsequently sputter-coated with a $3 \mathrm{~nm}$ thick layer of gold (80\%) / palladium (20\%) $(90 \mathrm{~s}, 45 \mathrm{~mA}$; Balzers SCD 030, Balzers, Liechtenstein) and analysed using cold field emission Scanning Electron Microscopy (SEM) (LEO 440, Electron Microscopy Ltd, Cambridge, United Kingdom).

\section{Statistical Analysis}

Statistical analysis was performed using SPSS 22.0 software for Windows (SPSS Inc., Chicago, IL, USA). Data were analyzed using Kaplan-Meier (Log Rank (Mantel-Cox)) tests to obtain the overall success, survival and failure rates in relation to observation time, and Wilcoxon test and McNemar test were used to test differences in the overall quality of survival. The alpha level was set at 0.05 in all tests. 


\section{RESULTS}

After 36 months no dropouts were experienced, 60 indirect posterior restorations (IDS, $n=30$; DDS, $n$ = 30) were evaluated. Mean observation time was 37.6 months (SD 2.9 months, min. 32 months, max. 43 months). Seventeen men and 13 women were included. The distribution of the location of the restorations is overviewed in table 1.

The overall success rate (Hickel score 1-3, restorations without any adverse effects, $n=50$ ) after 36 months is $85 \%$, not different for the IDS and DDS group ( $86.7 \%$ versus $83.3 \%, p=0.7$; KaplanMeier, Log Rank (Mantel-Cox), $\mathrm{Cl}=95 \%)$.

The overall survival rate (Hickel score $1-4, n=59$ ) is $98.3 \%$ after 36 months. The survival rates for IDS and DDS were $100 \%$ and $96.7 \%$ respectively (not significantly different, $p=0.32$; KaplanMeier, Log Rank (Mantel-Cox), Cl=95\%). Following Hickel criteria score 4 (table 5, figure 7) relative failures occurred in the DDS group $(n=5$; tooth chipping $n=3$, periodontal complications $n=1$ and fracture $n=1$ ) and in the IDS group ( $n=4$; periodontal complications $n=1$, secondary caries $n=1$, debonding $n=1$, excessive wear $n=1$ ). Tooth chipping with dentin exposure (figure 2 ) after 12 months was seen in a patient with severe teeth grinding (and scored again after 36 months in the same patient), the other chipping failure was a small fragment on an occlusal buccal cusp. Both periodontal complications (IDS and DDS) occurred in the same patient. The fracture (figure 3) originated after 36 months on a bearing cusp and part of the restoration. It could be repaired with a composite material. The debonding failure was a complete adhesive failure between the luting agent and the restoration and occurred 14 months after luting (figure 4). After cleaning the luting surface and removing the composite from the restoration surface, the restoration was replaced using the same adhesive protocol. Excessive wear was seen in a patient with severe teeth grinding (figure 5). In general, restorations scored a duller surface after 36 months compared to 1 week. Some patients $(n=5)$ experienced some postoperative sensitivity after 1 week, but this had resolved at 12 months. No patients reported tooth hypersensitivity after 36 months. 
One absolute failure had occurred in the DDS group (secondary caries, figure 6). The secondary caries developed in a medically compromised patient whose oral hygiene had seriously deteriorated, resulting in deep caries, imposing the prognosis of this tooth.

Patients did not call or come in for any kind of failure. All failures were noted at the planned follow-up moments.

\section{DISCUSSION}

In this clinical trial the performance of partial ceramic restorations bonded employing Immediate (IDS) or Delayed Dentin Sealing (DDS) in the same patient were compared. Based on non-significant differences in the success and survival rates and the quality of survival with IDS and DDS, the null hypothesis could not be rejected. The results cover up a period of 36 months. All absolute and relative failures were left unnoticed by the patient, hence were observed at the scheduled recall visit. Consequently, the exact time of occurrence could not be registered. According to the Hickel criteria 23 a score 4 or 5 is considered unacceptable and the restoration should be modified or remade. In this study, a score 4 is considered a relative failure due to the ability to repair the restoration and is defined as survival. Repaired restorations can be further monitored and evaluated. 24 A score 5 is considered an absolute failure due to the impossibility to repair this failure.

The number of absolute failures was limited; one restoration presented with secondary caries and had to be extracted. Two patients showed very poor oral hygiene despite regular adjustments during dental-checks ups every 6 months. Unfortunately this resulted in caries during follow-up. One tooth with secondary caries resulted in a relative failure and the other in an absolute failure. Depending on the location and the accessibility of the cavity caries results in an absolute or a relative failure. 24 Secondary caries develops mainly on the proximal gingival floor of class II restorations, usually being independent of the restorative material. 25-27 
Two relative failures occurred in one patient with severe teeth grinding. He reported not to have used the provided splint at night. The cohesive strength of ceramics proved insufficient for this (and possibly also others) individuals with parafunctional habits 28 and thus a splint is indicated for such patients. One failure in this patient being excessive wear and one being tooth chipping (figure 4 and 5). A possible explanation for the occurrence of tooth chipping is that the preparation design had not been uniform. The thickness of the ceramic was not similar all over the tooth, leading to a variation in wall height and resulting in a higher stress concentration. 29 This is also considered a probable cause of failure in figure 3 (right) because the SEM image shows an abrupt geometry variation; a very thick block of ceramic to a very thin part of ceramic on a very small distance. This results in an unfavorable stress distribution that could create a fracture site. In both failures in the patient with severe teeth grinding a fracture line is seen in the ceramic (figure 2 and 5) and received a score 3 for Hickel criteria on fracture of material and retention. Cracks are acceptable as long as there are no clinical symptoms present. 24 A small fracture in the ceramic is not always a problem as long as the location and adhesive is supportive and thus the stress can be distributed enough to prevent the restoration from catastrophic failure. 30 Especially in patients with severe teeth grinding, compromised design of the restoration or preparation is less forgiving. One of the relative failures was due to a fracture (figure 3). Here, the stress distribution may lead to a problem due to design of the restoration (thin isthmus). Because the mesio-lingual cusp was left too high and too thin, cuspal deflection may have lead to the fracture. Cuspal coverage is commonly recommended in order to protect the weakened tooth structure. 31 The benefit of a full cuspal coverage design (onlay) can be explained by the amount of the remaining tooth structure 32 , resulting in favorable distribution of stresses in teeth and reduces risk of fracture. 33 The discolored part of the wall indicates leakage of the restoration, probably due to cuspal deflection the bonding disrupted in this part. Preparation margins should have correct configuration to prevent chippings and cracks from and in the ceramic leading to crack propagation. 34 Fracture initiation sides are often created by parts in the restoration where the ceramic 
is very thin or where there is an air bubble present in the ceramic material. 29,34 Avoiding marginal ridge contact is recommended for these kind of restorations. In partial ceramic restorations IDS is thought to improve the adhesion resulting in improvement of the fracture strength. 35 Although we did not find any statistically significant difference in the performance of partial restorations using IDS or DDS some failures only occurred in the DDS group; tooth chipping and fractures. Further follow-up is needed to support the in vitro results and to see if IDS could indeed prevent some failures in partial indirect restorations on the mid- and long-term analysis.

In the debonding failure after 14 months (figure 4), the disto-buccal part of the restoration showed a discolored part which could indicate that there was not enough luting agent. Early failures are commonly related to technical flaws and not as a consequence of fatigue. During luting of this restoration, the cement was already partly light-cured by environment light, compromising the initial fit of the restoration. This part of the cement had to be removed and the procedure had to be redone. This procedure eventually resulted in insufficient marginal sealing.

Two periodontal failures were in a single patient as a result of poor oral hygiene during the follow-up period and to lack of regular dental check-ups. The increased pocket depths are not likely to have high impact on the longevity of the restoration itself, but rather reflects the functional oral environment. The restorations of this patient were not in direct contact with the periodontal tissues.

The survival rate of the restorations in this study (98.3\%) is comparable to that in other studies but the success rate $(85 \%)$ is somewhat lower than reported elsewhere. 3 The former may reflect the (initially limited) experience of the team in providing this type of restorations as training and experience is presumed to affect the outcome. No endodontic complications were seen while this is a common failure in other studies (3\%). 3 This finding reflects the minimal invasive preparation design as the amount of tooth structure reduction is considered to be an important factor affecting postoperative tooth sensitivity. 32 
Clinically it is difficult, to differentiate between gaps at the interface between luting material and hard tissues, and between luting material and restoration in compromised restorations. SEM examination was considered quite useful in assessing these aspects and is recommended for other clinical survival studies as well.

\section{CONCLUSIONS}

Adhesively luted, partial ceramic restorations in vital molar teeth have a good prognosis but do not benefit from IDS with respect to success and survival rates after 3 years of function.

\section{Acknowledgement}

The authors acknowledge Alette van Elk (TTL Oosterwijk Dental Laboratory Oosterwijk/Elysee, Groningen, The Netherlands) for her assistance in fabricating the ceramic inlays, as well as Berend Blok for all the work on wear analysis. The authors extend their gratitude to Ivoclar Vivadent (Schaan, Liechtenstein) and Kuraray (Osaka, Japan) for their generous provision of some of the materials used in this study.

\section{Conflict of interest}

The authors' institutions supported this study. The authors declare that they have no conflicts of interest and that they did not have any commercial interest in any of the materials used in this study. 


\section{References}

1. Thordrup M, Isidor F, Horsted-Bindslev P. A prospective clinical study of indirect and direct composite and ceramic inlays: Ten-year results. Quintessence Int 2006;37:139-144.

2. Pol CW, Kalk W. A systematic review of ceramic inlays in posterior teeth: An update. Int $\mathrm{J}$ Prosthodont 2011;24:566-575.

3. Morimoto S, Rebello de Sampaio FB, Braga MM, Sesma N, Ozcan M. Survival rate of resin and ceramic inlays, onlays, and overlays: A systematic review and meta-analysis. J Dent Res 2016;95:985994.

4. Conrad HJ, Seong WJ, Pesun IJ. Current ceramic materials and systems with clinical recommendations: A systematic review. J Prosthet Dent 2007;98:389-404.

5. McLaren EA, Whiteman YY. Ceramics: Rationale for material selection. Compend Contin Educ Dent 2010;31:666-8, 670, 672 passim; quiz 680, 700.

6. Aldegheishem A, loannidis G, Att W, Petridis H. Success and survival of various types of all-ceramic single crowns: A critical review and analysis of studies with a mean follow-up of 5 years or longer. Int $J$ Prosthodont 2017;30:168-181.

7. Pashley EL, Comer RW, Simpson MD, Horner JA, Pashley DH, Caughman WF. Dentin permeability: Sealing the dentin in crown preparations. Oper Dent 1992;17:13-20.

8. Paul SJ, Scharer P. The dual bonding technique: A modified method to improve adhesive luting procedures. Int J Periodontics Restorative Dent 1997;17:536-545.

9. Magne P. Immediate dentin sealing: A fundamental procedure for indirect bonded restorations. J Esthet Restor Dent 2005;17:144-54; discussion 155.

10. Magne P, Kim TH, Cascione D, Donovan TE. Immediate dentin sealing improves bond strength of indirect restorations. J Prosthet Dent 2005;94:511-519.

11. Breschi L, Mazzoni A, Ruggeri A, Cadenaro M, Di Lenarda R, De Stefano Dorigo E. Dental adhesion review: Aging and stability of the bonded interface. Dent Mater 2008;24:90-101.

12. Lee JI, Park SH. The effect of three variables on shear bond strength when luting a resin inlay to dentin. Oper Dent 2009;34:288-292.

13. Gresnigt MM, Cune MS, de Roos JG, Ozcan M. Effect of immediate and delayed dentin sealing on the fracture strength, failure type and weilbull characteristics of lithiumdisilicate laminate veneers. Dent Mater 2016;32:e73-81.

14. Qanungo A, Aras MA, Chitre V, Mysore A, Amin B, Daswani SR. Immediate dentin sealing for indirect bonded restorations. J Prosthodont Res 2016.

15. Choi YS, Cho IH. An effect of immediate dentin sealing on the shear bond strength of resin cement to porcelain restoration. J Adv Prosthodont 2010;2:39-45. 
16. Dalby R, Ellakwa A, Millar B, Martin FE. Influence of immediate dentin sealing on the shear bond strength of pressed ceramic luted to dentin with self-etch resin cement. Int J Dent 2012;2012:310702.

17. Duarte S,Jr, de Freitas CR, Saad JR, Sadan A. The effect of immediate dentin sealing on the marginal adaptation and bond strengths of total-etch and self-etch adhesives. J Prosthet Dent 2009;102:1-9.

18. Hu J, Zhu Q. Effect of immediate dentin sealing on preventive treatment for postcementation hypersensitivity. Int J Prosthodont 2010;23:49-52.

19. Kitayama S, Nasser NA, Pilecki P, Wilson RF, Nikaido T, Tagami J, Watson TF, Foxton RM. Effect of resin coating and occlusal loading on microleakage of class II computer-aided design/computeraided manufacturing fabricated ceramic restorations: A confocal microscopic study. Acta Odontol Scand 2011;69:182-192.

20. Leesungbok R, Lee SM, Park SJ, Lee SW, Lee do Y, Im BJ, Ahn SJ. The effect of IDS (immediate dentin sealing) on dentin bond strength under various thermocycling periods. J Adv Prosthodont 2015;7:224-232.

21. Magne P, So WS, Cascione D. Immediate dentin sealing supports delayed restoration placement. J Prosthet Dent 2007;98:166-174.

22. Oliveira L, Mota EG, Borges GA, Burnett LH,Jr, Spohr AM. Influence of immediate dentin sealing techniques on cuspal deflection and fracture resistance of teeth restored with composite resin inlays. Oper Dent 2014;39:72-80.

23. Hickel R, Peschke A, Tyas M, Mjor I, Bayne S, Peters M, Hiller KA, Randall R, Vanherle G, Heintze SD. FDI world dental federation: Clinical criteria for the evaluation of direct and indirect restorationsupdate and clinical examples. Clin Oral Investig 2010;14:349-366.

24. Hickel R, Roulet JF, Bayne S, Heintze SD, Mjor IA, Peters M, Rousson V, Randall R, Schmalz G, Tyas M, Vanherle G. Recommendations for conducting controlled clinical studies of dental restorative materials. science committee project 2/98--FDI world dental federation study design (part I) and criteria for evaluation (part II) of direct and indirect restorations including onlays and partial crowns. J Adhes Dent 2007;9 Suppl 1:121-147.

25. Mjor IA. Frequency of secondary caries at various anatomical locations. Oper Dent 1985;10:88-92.

26. Mjor IA. The location of clinically diagnosed secondary caries. Quintessence Int 1998;29:313-317.

27. Mjor IA, Qvist V. Marginal failures of amalgam and composite restorations. J Dent 1997;25:25-30.

28. Friedman MJ. A 15-year review of porcelain veneer failure--a clinician's observations. Compend Contin Educ Dent 1998;19:625-8, 630, 632 passim; quiz 638.

29. Rekow ED, Zhang G, Thompson V, Kim JW, Coehlo P, Zhang Y. Effects of geometry on fracture initiation and propagation in all-ceramic crowns. J Biomed Mater Res B Appl Biomater 2009;88:436446. 
30. de Kok P, Pereira GKR, Fraga S, de Jager N, Venturini AB, Kleverlaan CJ. The effect of internal roughness and bonding on the fracture resistance and structural reliability of lithium disilicate ceramic. Dent Mater 2017;33:1416-1425.

31. Kuijs RH, Fennis WM, Kreulen CM, Roeters FJ, Verdonschot N, Creugers NH. A comparison of fatigue resistance of three materials for cusp-replacing adhesive restorations. J Dent 2006;34:19-25.

32. Edelhoff D, Sorensen JA. Tooth structure removal associated with various preparation designs for posterior teeth. Int J Periodontics Restorative Dent 2002;22:241-249.

33. Mondelli J, Steagall L, Ishikiriama A, de Lima Navarro MF, Soares FB. Fracture strength of human teeth with cavity preparations. J Prosthet Dent 1980;43:419-422.

34. Mecholsky JJ,Jr. Fractography: Determining the sites of fracture initiation. Dent Mater 1995;11:113-116.

35. van den Breemer CRG, Ozcan M, Cune MS, van der Giezen R, Kerdijk W, Gresnigt MMM. Effect of immediate dentine sealing on the fracture strength of lithium disilicate and multiphase resin composite inlay restorations. J Mech Behav Biomed Mater 2017;72:102-109.

\section{Captions to the tables and figures:}

\section{Tables:}

Table 1 Distribution of restored teeth and extension of the restorations in the maxilla and mandible in the test (Immediate Dentin Sealing-IDS) and control (Delayed Dentin Sealing-DDS) group.

Table 2 The brands, types, manufacturers, chemical compositions and batch numbers of the major materials used in this study.

Table 3a-b Clinical protocol for the a) test group (Immediate Dentin Sealing-IDS), b) control group (Delayed Dentin Sealing-DDS).

Table 4 Clinical protocol for luting procedures of the ceramic restorations.

Table 5 Summary of Hickel criteria evaluation at 1 week, at 12 months and at 36 months.

\section{Figures:}

Figure 1 Consolidated Standards of Reporting Trials (CONSORT) 2010 flow diagram. 
Figure 2 Images of tooth chipping with dentin exposure.

Figure 3 Images of fracture failure.

Figure 4 Images of debonding failure.

Figure 5 Images of excessive wear of the ceramic and fracture lines in the ceramic.

Figure 6 X-ray presenting secondary caries.

Figure 7 Kaplan-Meier curve of the relative failures of partial ceramic restorations bonded employing either Immediate (IDS) or Delayed Dentin Sealing (DDS) (IDS: 100\%, $n=30$, events $n=4$; DDS: $96.7 \%, n=30$, events $n=5$ ). 


\begin{tabular}{|c|c|c|c|c|c|c|}
\hline Test group & Molars (n) & & & & & Total (N) \\
\hline & 0 cusps replaced & 1 cusp replaced & 2 cusps replaced & 3 cusps replaced & 4 cusps replaced & \\
\hline Maxilla (n) & 2 & 4 & 5 & 2 & 6 & 19 \\
\hline Mandible (n) & 2 & 1 & 1 & 4 & 3 & 11 \\
\hline Total (N) & 4 & 5 & 6 & 6 & 9 & 30 \\
\hline
\end{tabular}

\begin{tabular}{|c|c|c|c|c|c|c|}
\hline Control group & Molars (n) & & & & & Total (N) \\
\hline & 0 cusps replaced & 1 cusp replaced & 2 cusps replaced & 3 cusps replaced & 4 cusps replaced & \\
\hline Maxilla (n) & 5 & 1 & 1 & 2 & 5 & 14 \\
\hline Mandible (n) & 2 & 3 & 5 & 2 & 4 & 16 \\
\hline Total (N) & 7 & 4 & 6 & 4 & 9 & 30 \\
\hline
\end{tabular}

Table 1. Distribution of restored teeth and extension of the restorations in the maxilla and mandible in the test (Immediate Dentin Sealing-IDS) and control (Delayed Dentin Sealing-DDS) group.

\begin{tabular}{|c|c|c|c|c|}
\hline Brand & Type & Nanufasturer & Chemical composition & Batch-numbar. \\
\hline TPS-6maxpress & Pressade ceramic & Troctar Vivadent, Schaan, Lachorastain & 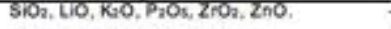 & $\overline{-}$ \\
\hline Variolink Uiba Catalyst $/$ Base & Dual-cure luting composhe & trociar Vivadent & 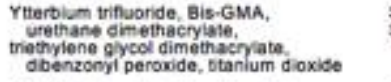 & $\begin{array}{l}\$ 27220 i \\
\$ 06646\end{array}$ \\
\hline Clearti SE Primer & Adnesive primer & Kuraray CO., Lid.. Osaika, Japan & $\begin{array}{l}\text { HEMA, hydrophillc dimethacryiate. } \\
\text { water, photo-nitiator }\end{array}$ & 200022 \\
\hline Clearti SE Bond & Adresive resin & Kuraray $\mathrm{CO}_{4}$ Lte. & $\begin{array}{l}\text { MOP. HEMA, bis-GMA, hydrophilic } \\
\text { dimethacrylase, water, } \\
\text { photo initiator, silanatiod coloidal } \\
\text { silica }\end{array}$ & 2T003 \\
\hline Excite F DSC & Single component adhesive & troclar Vivadent & 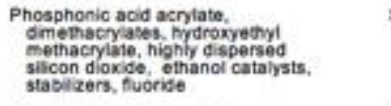 & $\$ 36470$ \\
\hline Covet-sand & Blast.coating ageont & 3M ESPE, Minn, USA & $\begin{array}{l}\text { Aluminimm triaxide particles } \\
\text { Cosited with silca, } 30 \text { jm }\end{array}$ & $\begin{array}{l}4463177 \\
534151\end{array}$ \\
\hline ESPE-SII & Sliane coupling apent & 3M ESPE & $\begin{array}{l}\text { Ethyl alcohol, 3-methacryloxypropyt } \\
\text { timmethoxysliane, othanol }\end{array}$ & 518272 \\
\hline Monobond Plus & One component primer & trociar Vivadent & $\begin{array}{l}\text { Ethanol, 3-trimegherysisylproget } \\
\text { meethacilate, mothacrylated phosphoric } \\
\text { actid estor }\end{array}$ & $\$ 31153$ \\
\hline IPS Ceramic ench & Hyerohuoric acid & trociar Vivadent & <5\% Hydrofluoric acid & $\$ 26140$ \\
\hline IPS Neutralizing powser & Dowder & trociar Vivadent & Sodium eartsonate & $\$ 34285$ \\
\hline Ultra-ebeh & 35\% Phosporic acis & Ultradent, South Jortan UT, USA & $35 \%$ phosphorie acid & 130910 \\
\hline Clearta Majesty Flow & Phoso-cure composite & Kuraray co. & 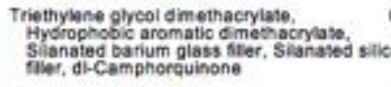 & $\begin{array}{l}\text { O03398A } \\
\text { ca }\end{array}$ \\
\hline Glycerin gel & K.Y. lubricating get & K-Y, Johnson \& Johnson, Sezanne, France & 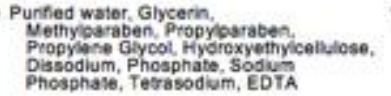 & $1233 \mathrm{~V}$ \\
\hline
\end{tabular}

Table 2. The brands, types, manufacturers, chemical compositions and batch numbers of the materials used. 


\subsection{Tooth preparation}

1.2 Apply Clearfil SE Primer, active brushing motion

1.3 Air suction

1.4 Apply Clearfil SE Adhesive, active brushing motion 10

1.5 Air-thin 10

$\begin{array}{ll}\text { 1.6 Photo-polymerize } & 10\end{array}$

1.7 Apply flowable resin (Clearfil Majesty Flow)
1.8 Photo-polymerize

1.9 Apply glycerin gel
1.10 Photo-polymerization at buccal, oral and proximal sites 40 each

1.11 Rinse until clean surface

1.12 Clean the enamel outline with a rubber-point or a bur

1.13 Make impression

Visit 2: Cleaning and conditioning of the tooth prior to luting

2.1 Clean tooth surface (EMS)

2.2 Silica-coat (CoJet-sand) the IDS $2-3$

2.3 Acid etch enamel $\quad 30$

2.4 Rinse $\quad 30$

2.5 Dry

2.6 Apply silane (ESPE-Sil) on the IDS $\quad 60$

2.7 Apply adhesive resin (Excite F DSC)

2.8 Apply resin cement (Variolink Ultra) on the tooth

2.9 Place the partial restoration onto the tooth

2.10 Remove excess cement

2.11 Photo-polymerize at buccal, oral and proximal sides 40 each

2.12 Apply glycerin gel

2.13 Photo-polymerize at buccal, oral and proximal sides 40 each

Table 3-a. Clinical protocol for the test group (Immediate Dentin Sealing-IDS). 


\subsection{Tooth preparation}

1.2 Make impression

Visit 2: Cleaning and conditioning of the tooth prior to luting

2.1 Clean tooth surface (EMS)

2.2 Acid etch enamel and dentin

2.5 Apply adhesive resin (Excite F DSC)

2.6 Apply resin cement (Variolink Ultra) on the tooth

2.7 Place the partial restoration onto the tooth

2.8 Remove excess cement

2.9 Photo-polymerize at buccal, oral and proximal sides

40 each

2.10 Apply glycerin gel

2.11 Photo-polymerize at buccal, oral and proximal sides

40 each

Table 3-b. Clinical protocol for the control group (Delayed Dentin Sealing-DDS).

Visit 2: Luting procedures of the ceramic restorations

2.1. Apply hydrofluoric acid etch (IPS Ceramic etch)

2.2. Rinse and neutralize

2.3. Rinse and dry

2.4. Ultrasonically clean in distilled water

2.5. Dry

2.6. Apply silane (Monobond Plus) one coat and wait its reaction

2.7. Apply adhesive resin (Excite F DSC)

2.8. For the subsequent procedures, follow step 2.8 in Table $3 a$ or step 2.6 in Table 3b.

20

60

300

60

0

0
Working time (s)

Table 4. Clinical protocol for luting procedures of the ceramic restorations. 


\begin{tabular}{|c|c|c|c|c|c|c|c|}
\hline Hickel - criteria & & $\begin{array}{r}\text { IDS } \\
1 \text { week }\end{array}$ & IDS & 36 months & \multicolumn{2}{|l|}{ DDS } & 36 months \\
\hline A. Esthetic Properties & & & & & & & \\
\hline 1. Surface lustre & 1 & 29 & 13 & 9 & 29 & 9 & 7 \\
\hline & 2 & 1 & 10 & 14 & 1 & 11 & 13 \\
\hline & 3 & - & 7 & 7 & - & 10 & 10 \\
\hline & 4 & - & - & - & - & - & \\
\hline & 5 & - & - & t & - & - & \\
\hline 2. Staining surface / margin & 1 & 30 & 28 & 21 & 30 & 27 & 21 \\
\hline & 2 & - & 1 & 7 & - & 2 & 8 \\
\hline & 3 & - & 1 & 2 & - & 1 & 1 \\
\hline & 4 & - & . & . & - & - & \\
\hline & 5 & - & . & t. & - & . & \\
\hline 3. Color match and translucency & 1 & 24 & 21 & 20 & 28 & 26 & 23 \\
\hline & 2 & 5 & 5 & 6 & 1 & 1 & 4 \\
\hline & 3 & 1 & 4 & 4 & 1 & 3 & 3 \\
\hline & 4 & - & - & - & - & - & \\
\hline & 5 & - & - & . & - & - & - \\
\hline 4. Esthetic anatomical form & 1 & 30 & 30 & 30 & 30 & 30 & 30 \\
\hline & 2 & - & - & $f$ & - & - & \\
\hline & 3 & - & . & . & - & - & \\
\hline & 4 & - & - & t & - & - & \\
\hline & 5 & - & - & . & - & . & \\
\hline B. Functional properties & & & & & & & \\
\hline 5. Fracture of material and retention & 1 & 28 & 26 & 27 & 30 & 29 & 26 \\
\hline & 2 & 2 & 2 & . & - & - & 2 \\
\hline & 3 & - & 1 & 3 & - & 1 & \\
\hline & 4 & - & . & . & - & . & 2 \\
\hline & 5 & - & 1 & t & - & - & \\
\hline 6. Marginal adaptation & 1 & 26 & 23 & 17 & 28 & 20 & 16 \\
\hline & 2 & 3 & 7 & 12 & 2 & 9 & 13 \\
\hline & 3 & 1 & . & 1 & - & 1 & 1 \\
\hline & 4 & - & - & . & - & - & \\
\hline & 5 & - & . & t & . & . & 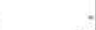 \\
\hline 7. Occlusal contour wear & 1 & 30 & 29 & 28 & 30 & 29 & 29 \\
\hline qualatitively / quantitatively & 2 & - & - & 1 & - & - & - \\
\hline & 3 & - & 1 & t. & - & 1 & 1 \\
\hline & 4 & - & . & 1 & - & - & \\
\hline & 5 & - & - & . & - & . & - \\
\hline 8. Approximal anatomical & 1 & - & 30 & 30 & - & 30 & 30 \\
\hline form contact point / contour & 2 & - & . & f & . & - & \\
\hline & 3 & - & - & . & - & - & \\
\hline & 4 & . & . & . & - & . & \\
\hline & 5 & - & - & f & - & . & 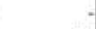 \\
\hline 9. Radiographic examination & 1 & 29 & 29 & 28 & 28 & 29 & 29 \\
\hline & 2 & 1 & 1 & 2 & 2 & 1 & 1 \\
\hline & 3 & - & - & - & - & - & \\
\hline & 4 & - & - & . & - & - & \\
\hline & 5 & . & - & . & - & - & . \\
\hline 10. Patient's view & 1 & 25 & 30 & 30 & 26 & 30 & 29 \\
\hline & 2 & - & - & f & - & - & - \\
\hline & 3 & 5 & . & t & 4 & - & \\
\hline & 4 & . & . & t & - & - & 1 \\
\hline & 5 & - & - & . & - & - & \\
\hline C. Biological properties & & & & & & & \\
\hline 11. Postoperative (hyper-)sensitivity & 1 & 28 & 30 & 30 & 27 & 30 & 30 \\
\hline and tooth vitality & 2 & 1 & . & f & 3 & . & \\
\hline & 3 & 1 & - & -1 & - & - & \\
\hline & 4 & . & . & . & . & . & \\
\hline & 5 & - & - & . & - & - & . \\
\hline 12. Recurrence of caries, erosion, & 1 & 30 & 30 & 28 & 30 & 30 & 27 \\
\hline abfraction & 2 & . & - & - & - & . & 1 \\
\hline & 3 & - & . & 1 & - & - & 1 \\
\hline & 4 & - & . & . & - & . & \\
\hline & 5 & . & . & 1 & - & . & 1 \\
\hline 13. Tooth integrity (enamel cracks, & 1 & 29 & 30 & 30 & 30 & 29 & 28 \\
\hline tooth fractures) & 2 & 1 & - & ${ }^{-1}$ & - & - & \\
\hline & 3 & - & - & . & - & - & - \\
\hline & 4 & - & - & t. & - & 1 & 2 \\
\hline & 5 & - & - & . & - & - & . \\
\hline 14. Periodontal response (compared to & 1 & 18 & 19 & 18 & 20 & 19 & 16 \\
\hline a reference tooth) & 2 & 12 & 7 & 9 & 0 & 8 & 12 \\
\hline & 3 & . & 3 & 3 & - & 2 & 2 \\
\hline & 4 & . & 1 & f & . & 1 & \\
\hline & 5 & - & . & t & - & . & . \\
\hline 15. Adjacent mucosa & 1 & 23 & 30 & 28 & 26 & 30 & 30 \\
\hline & 2 & 7 & - & 2 & 4 & . & \\
\hline & 3 & - & . & 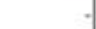 & . & . & \\
\hline & 4 & - & - & . & - & - & \\
\hline & 5 & - & . & t & - & - & . \\
\hline 16. Oral and general health & 1 & 30 & 30 & 30 & 30 & 30 & 30 \\
\hline & 2 & - & - & . & - & - & \\
\hline & 3 & - & - & -1 & - & - & \\
\hline & 4 & - & - & t & - & - & \\
\hline & 5] & . & . & 与 & - & - & \\
\hline
\end{tabular}

Table 5. Summary of the Hickel criteria evaluation at 1 week, at 12 months and at 36 months. Restorations with Hickel score 1-3 are considered to have succeed. Restorations with Hickel score 4 
are considered to have relatively failed and are considered to have survived. Restorations with Hickel score 5 are considered to have absolutely failed.

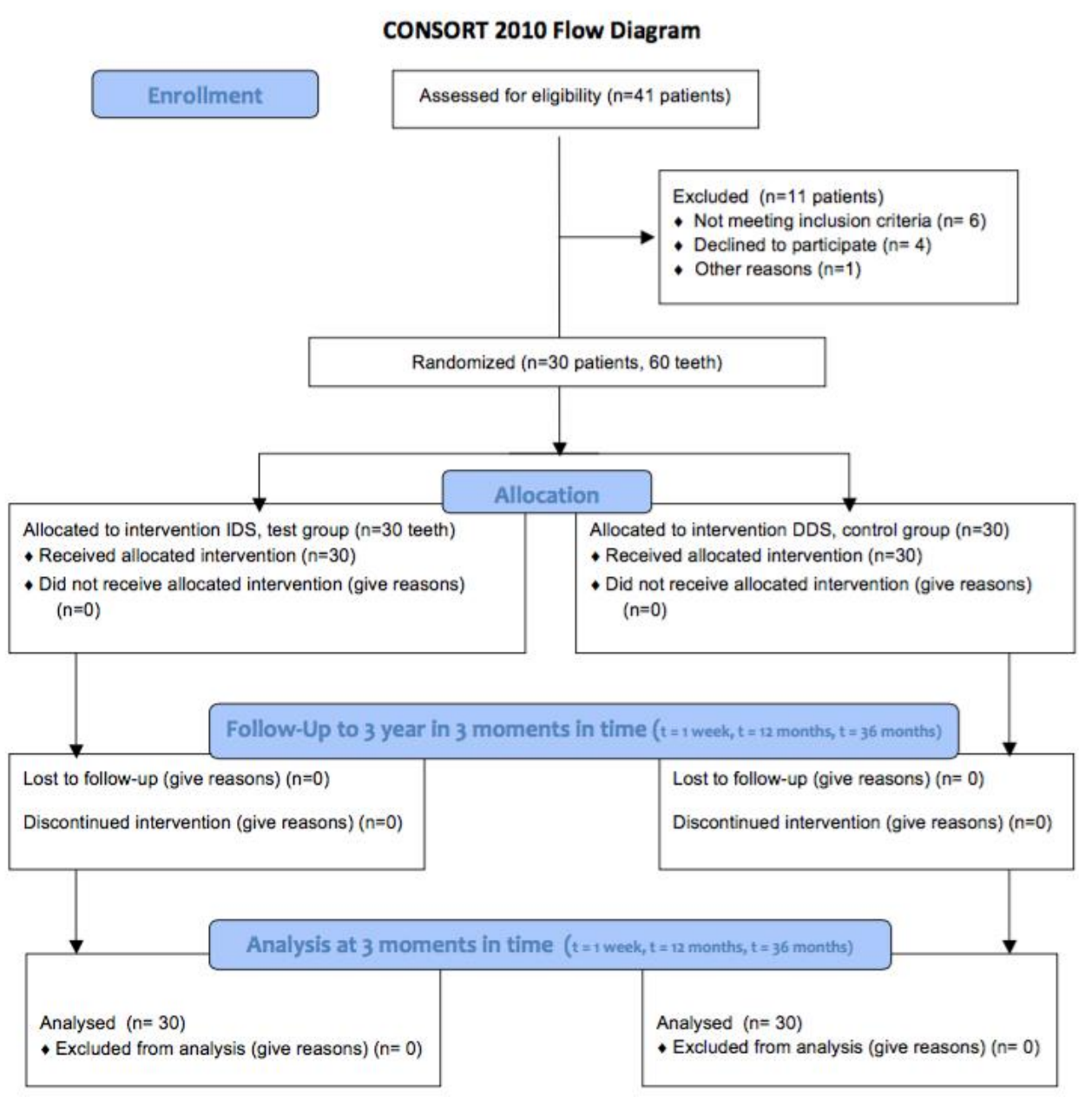

Fig. 1 Consolidated Standards of Reporting Trials (CONSORT) 2010 flow diagram explaining enrollment, intervention allocation, follow-up and data analysis. 

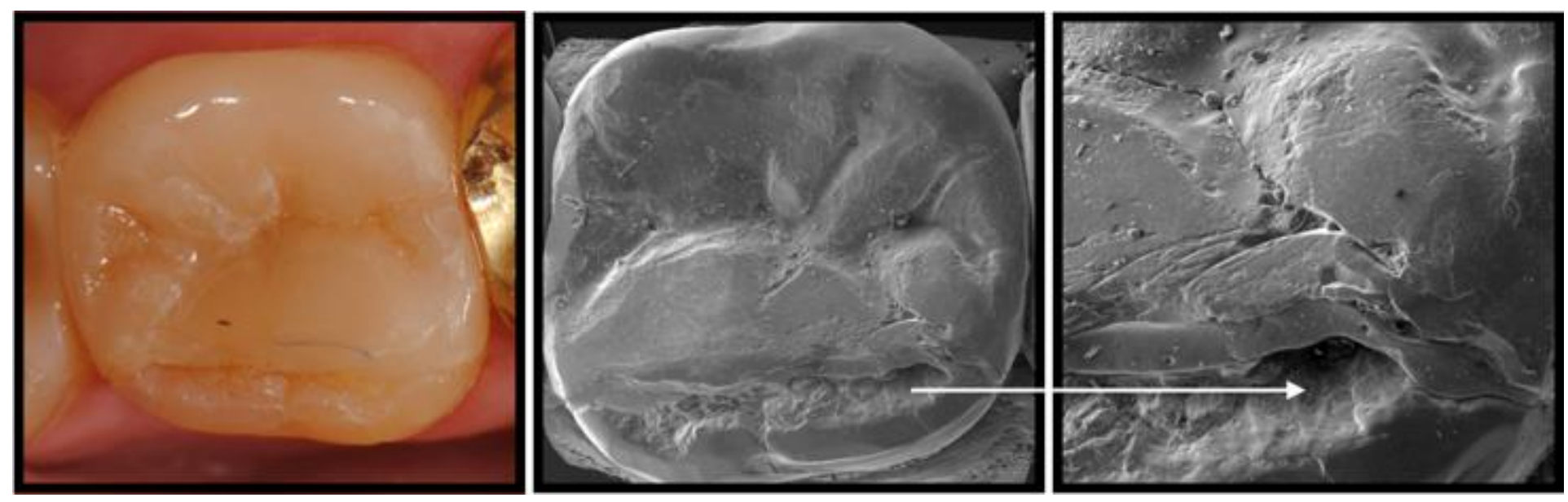

Fig. 2 (left) Chipping of tooth 37 with dentin exposure. (middle) SEM image of chipping of tooth 37 with dentin exposure. Note the fracture line from buccal to the mesial part. (right) Detailed SEM image of the chipping plane. Note the fracture line in the ceramic.
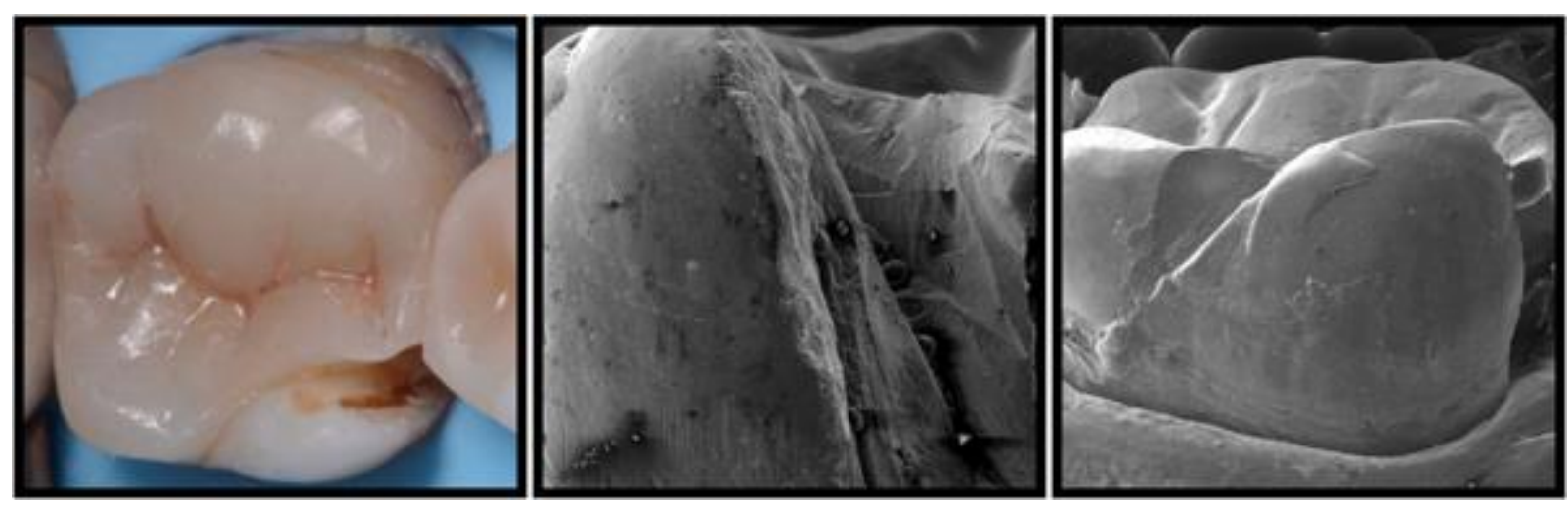

Fig. 3 (left) Fracture of the mesio-lingual aspect of tooth 36. Note the discolored dentin part at the inner wall next to a remnant of composite material (middle) SEM image of the fracture with the bearing intact cusp on the left side. Fracture visible in the ceramic with a part of the composite still attached to the tooth (right) SEM image of the lingual side of the tooth with the fracture in the ceramic on the mesio-lingual side. 


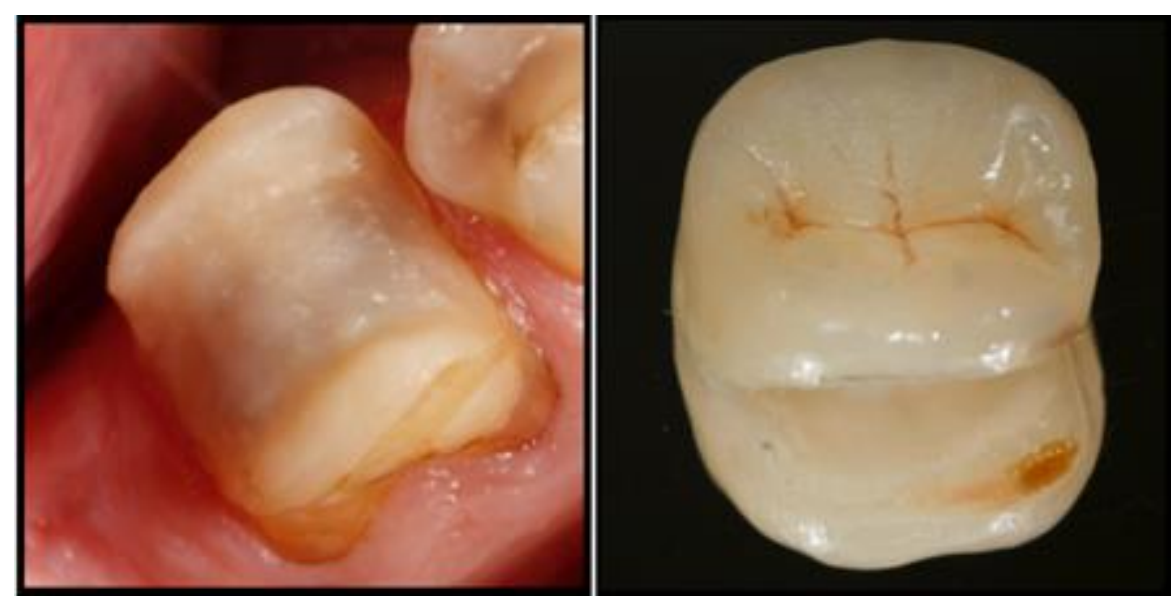

Fig. 4 (left) Tooth surface after debonding of restoration 36, note the intact IDS/composite layer. (right) Restoration debonding surface. Note the discolored disto-buccal part of the restoration.
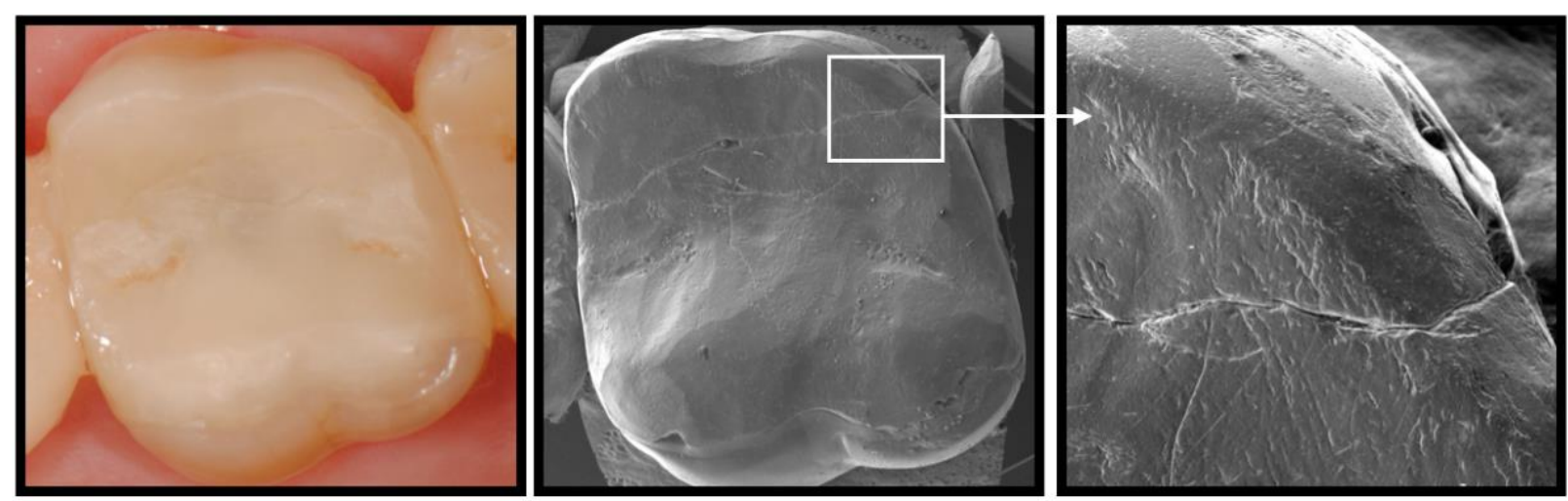

Fig. 5 (left) Excessive wear on the occlusal part of the restoration 26. Note the fracture line in the ceramic on the buccal side of the restoration. (middle) SEM image of restoration 26. Note the excessive wear and the clear fracture line in the ceramic from mesial to distal. (right) Detailed SEM image of small bending and branching of the fracture line in the ceramic. 


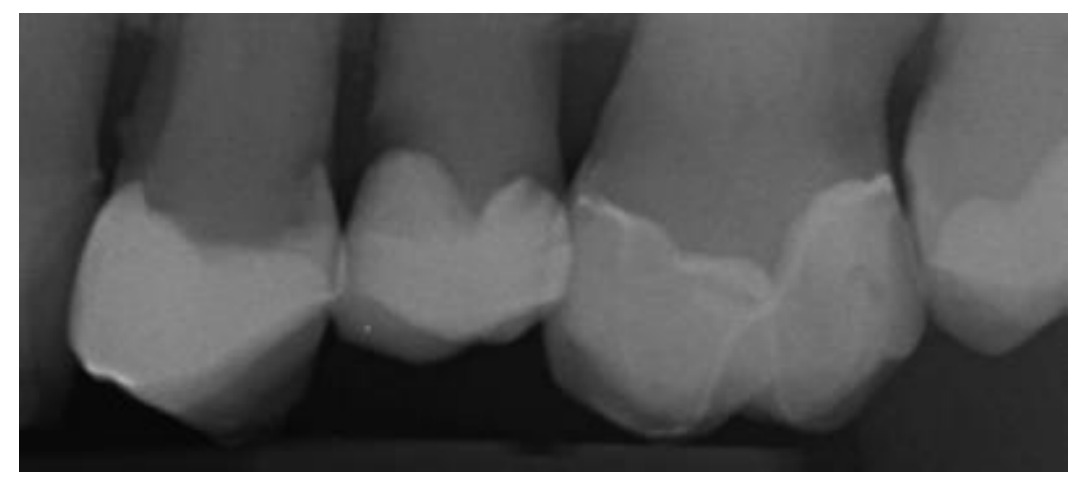

Fig. 6 Secondary caries at tooth 26, distal aspect. The lesion prooved an understatement of the intra-oral size of the cavity. Note also the secondary caries on teeth nrs. 24 and 35.

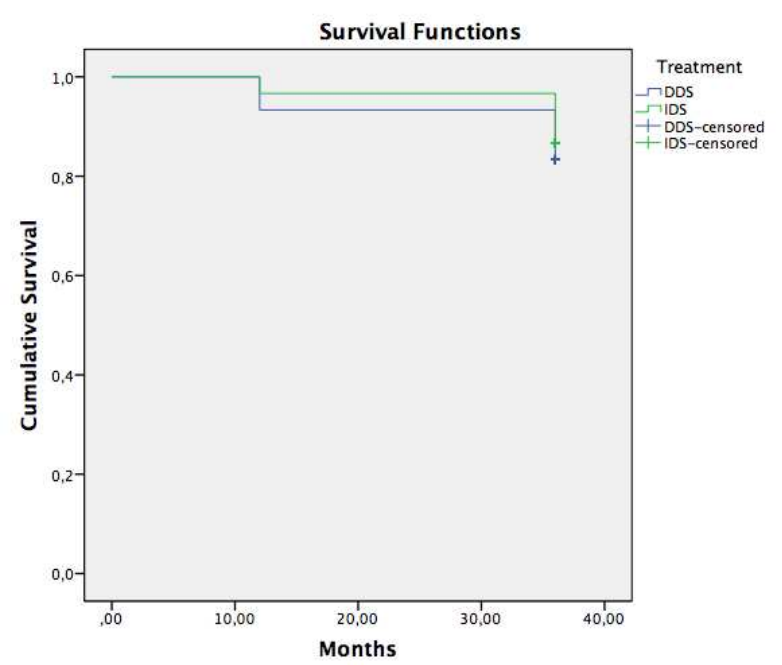

Fig. 7 Kaplan-Meier curve of the relative failures of partial ceramic restorations bonded employing either Immediate (IDS) or Delayed Dentin Sealing (DDS) (IDS: $86.7 \%, \mathrm{n}=30$, events $n=4$; DDS: $83.3 \%, n=30$, events $n=5$ ). 